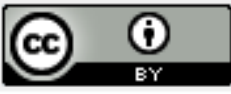

\title{
ANÁLISE ECONÔMICO-FINANCEIRA DA UTILIZAÇÃO DO GN EM SISTEMAS DE COGERAÇÃO APLICADOS A INSTALAÇÕES PREDIAIS
}

\section{ECONOMIC-FINANCIAL ANALYSIS FOR NG OPERATION OF COGENERATION SYSTEMS IN BUILDINGS}

\author{
Márcia Ferreira Cristaldo \\ Universidade Federal de Mato Grosso do Sul - UFMS \\ Centro de Ciências Exatas e Tecnologia - CCEP \\ Departamento de Engenharia Elétrica - DEL \\ Rua Herbert Moses, 429 Jardim Paulista CEP 79050-150 \\ Campo Grande - MS \\ 67-33429935/67-84210154 \\ mferreiracristaldo@gmail.com \\ Jeferson Meneguin Ortega \\ Engenheiro Eletrecista \\ Universidade Federal de Mato Grosso do Sul - UFMS \\ Centro de Ciências Exatas e Tecnologia - CCEP \\ Departamento de Engenharia Elétrica - DEL \\ Caixa Postal 549 \\ 79070-900 - Campo Grande - MS \\ 67-33457470/3345-7464 \\ jmortega@del.ufms.br
}




\title{
RESUMO
}

Sistemas de cogeração podem ampliar a capacidade instalada para geração de energia no Brasil. Podem também aumentar a confiabilidade do sistema de transmissão, diversificando a matriz energética e ampliando as oportunidades de negócio para o capital privado no mercado brasileiro. O Brasil apresenta expressivo potencial para a implantação de pequenas plantas de cogeração, sobretudo no setor de serviços (terciário), onde se concentra um número considerável de pequenos estabelecimentos comerciais com necessidades de energia elétrica, vapor, água quente e/ou água gelada (para refrigeração). Neste trabalho é apresentada uma metodologia de apoio aos novos usuários desse setor, a qual permite a análise da viabilidade econômico-financeira da utilização do gás natural em sistemas de cogeração como alternativa à eletricidade. Esta metodologia é baseada na técnica de dinâmica de sistemas, que permite tanto a representação do comportamento dinâmico das variáveis envolvidas, como a análise dos efeitos da competição dessas duas fontes de energia (gás natural e energia elétrica) junto ao mercado consumidores.

Palavras-Chaves: gás natural, cogeração, instalações prediais, dinâmica de sistemas.

\begin{abstract}
Cogeneration systems must increase the installed brazilian power generation capacity. It can also improve the reliability of the transmission system, using different energy sources in new power plants and enlarging the business opportunities for private capital in the brazilian market. Brazil presents expressive potential for distributed generation plants of small capacities, most of them in services sector (tertiary), where there is a considerable number of small companies needing electricity, steam, hot water and/or cold water (for refrigeration). In this work it is presented a methodology to support new users in that sector, which allows to analyze the economic-financial viability in the use of the natural gas as an alternative to electricity by using cogeneration systems. That methodology is based on the technique of systems dynamics, therefore in such a way allows the dynamic behavior representation of the involved variables, as it analyzes the effects due to the competition of natural gas and electric energy in the consumers market.
\end{abstract}

Keywords: natural gas, cogeneration, building facilities, systems dynamics.

\section{INTRODUÇÃO}

Nos últimos anos e em todo o mundo, a desregulamentação da indústria de energia elétrica tem levado a mudanças profundas na indústria e em seu mercado (LORA \& HADDAD, 2006, p.12). 
Segundo dados da ANEEL, o aumento médio em 2006 atingiu 5,98\% em relação à média de 2005, quando atingiram 19,93\%. Em 2004 os aumentos somaram 18,07\%, em 2003 atingiram $16,85 \%$ e $16,41 \%$ em 2002.

O Estado de Mato Grosso do Sul tem a maior tarifa do País e a sexta mais cara do mundo, superando a cobrada em países como Estados Unidos e México. Logo, o crescimento do Estado fica vulnerável, visto que este crescimento implica em maior demanda por energia elétrica. Consequentemente, na busca da expansão econômica, torna-se essencial investir em outros setores de fonte de energia, a exemplo dos sistemas de cogeração operando com gás natural.

A cogeração consiste na produção simultânea de energia térmica e energia elétrica a partir do uso de um combustível convencional (gás natural, óleo combustível, diesel e carvão) ou algum tipo de resíduo industrial (madeira, bagaço de cana, casca de arroz, etc.). $\mathrm{Na}$ cogeração pode-se alcançar um aproveitamento de até $80 \%$ de energia contida no combustível. Esta energia pode ser transformada em vapor, eletricidade, força motriz e frio, sendo ainda possível a produção de gás carbônico $(\mathrm{CO} 2)$ a partir da descarga dos gases de combustão (http://www.br.com.br).

A cogeração é aplicável em instalações que necessitem simultaneamente de energia térmica elétrica, em diversos segmentos, a exemplo dos seguintes potenciais clientes deste processo: Indústria (alimentos, bebidas, química, petroquímica, papel e celulose, cerâmica, farmacêutica), Comércio (shopping centers, supermercados, complexos comerciais) e serviços (hospitais, hotéis, aeroportos, grandes condomínios), sendo este último o chamado setor terciário. Podem ser apontadas as seguintes vantagens da cogeração: auto-suficiência energética, qualidade da energia elétrica, melhoria da eficiência energética do processo, redução dos impactos ambientais e foco na atividade principal da empresa.

Sistemas de cogeração apresentam uma alta eficiência, mas geralmente são projetados para atender apenas indústrias de médio e grande porte. Entretanto, estes sistemas tentam buscar um mercado competitivo, inovador e voltado para os consumidores, onde os negócios apenas têm êxito se focados no interesse destes clientes finais. Tal contexto enfatiza a confiabilidade, o aumento na eficiência energética, do desempenho ambiental e a prestação de serviços que atendam a outras necessidades da comunidade em geral. Associando-se estas transformações, em parte como causa, em parte como efeito, os avanços tecnológicos têm posicionado favoravelmente o sistema de cogeração frente aos grandes sistemas centralizados. 


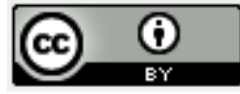

Os novos desenvolvimentos em tecnologias de geração termoelétrica em pequena escala, considerando motores alternativos, turbinas e microturbinas a gás, em um cenário de curto e médio prazo, têm colocado estas centrais como alternativa concreta de suprimento de energia elétrica e térmica, efetuando-se a geração de energia no ponto de consumo final (ou próximo deste).

O Brasil apresenta expressivo potencial para a implantação de pequenas plantas de cogeração, sobretudo no setor terciário, onde se concentra um número considerável de pequenos estabelecimentos comerciais com necessidades de energia elétrica, vapor, água quente e/ou água gelada (para refrigeração). $\mathrm{O}$ alto custo da eletricidade (ou energia elétrica), associado ao crescimento significativo no consumo desta fonte de energia, abre caminho para a instalação de centrais de cogeração que atendam necessidades elétricas e térmicas de comércio.

De acordo com Ortegosa (2006), o gás natural tornou-se, ao longo dos anos, uma excelente alternativa para uso em residências em função das inúmeras vantagens como a minimização de custos e melhoria da produtividade, que apresenta em relação ao GLP e a energia elétrica.

No Mato Grosso do Sul a empresa de distribuição de gás natural tem investido na aplicação de ramais de distribuição, de maneira a disponibilizar esta fonte de energia aos mais diferentes tipos de consumidores. Consequiêntemente, o combustível estará acessível em pelo menos $50 \%$ da área central alcançando o consumidor residencial, que poderá utilizar o combustível, por exemplo, como substituto do GLP (gás de cozinha) (MSGÁS, 2007). Com isso, o fornecimento de gás natural em instalações prediais é um dos mercados em grande desenvolvimento, principalmente, no Estado de Mato Grosso do Sul. Assim, este projeto de expansão da rede de distribuição contribui de modo efetivo ao uso desta fonte de energia, eliminando uma das barreira para a utilização do gás natural.

Neste contexto, este trabalho propõe uma metodologia de apoio aos novos usuários do setor predial residencial e comercial, que permita analisar a viabilidade econômico-financeira da utilização do gás natural em alternativa à energia elétrica, através do uso de sistemas de cogeração. A opção por sistemas de cogeração, de modo geral, é definida por condicionantes estritamente econômicas, adotada somente quando se observam reduções substanciais nos custos de energia. Na análise financeira, são consideradas as seguintes variáveis: custos de instalação, operação e manutenção, tarifas de energia elétrica (atuais e futuros), preço e 
disponibilidade do combustível, incentivos fiscais e retorno financeiro.

\section{ANALISE DA TARIFA}

A estratégia governamental de aumentar a participação do GN na matriz energética brasileira, implica em investimentos tanto na ampliação da produção nacional de gás natural (nas bacias de Santos-SP, Campos-RJ e no estado do Espírito Santo), como em infra-estrutura de transporte e distribuição capaz de disponibilizar o gás aos setores de consumo.

Neste sentido, o governo federal implementou o PAC - Programa de Aceleração do Crescimento, o qual deverá aplicar, no período de 2007-2010, um total de investimentos em infra-estrutura no valor de $\mathrm{R} \$ 503,9$ bilhões (DIEESE, 2007). Uma das áreas mais beneficiadas com os investimentos é o setor de fonte de energia, o qual deverá receber cerca de $45,5 \%$ destes recursos.

Na Figura 1 tem-se um gráfico mostrando o cresimento anual do gás natural na matriz energética brasileira.

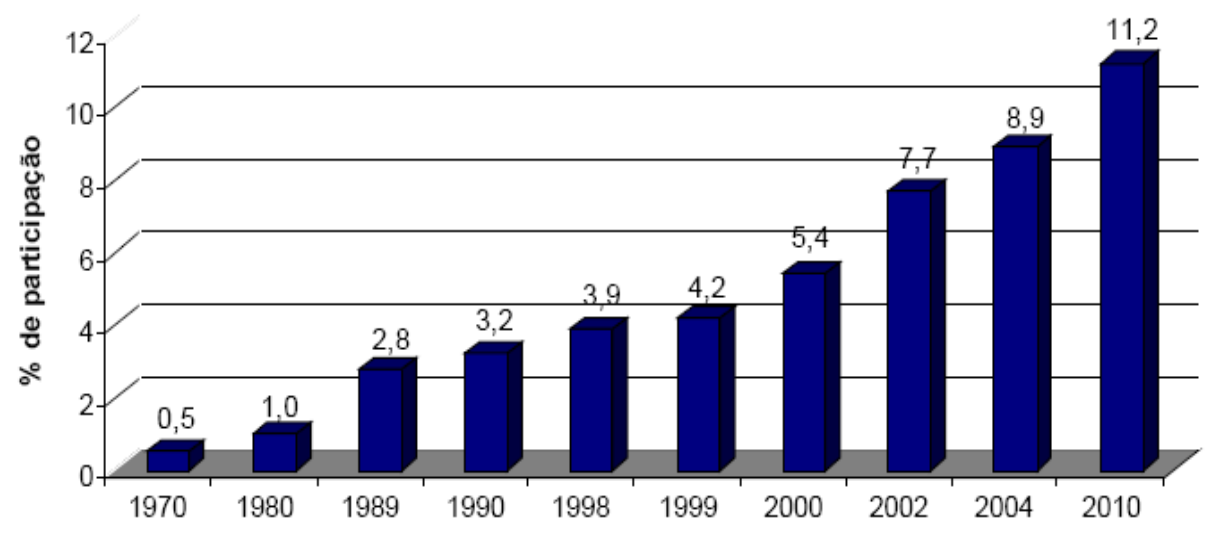

Figura 1 - Gráfico da matriz energética brasileira

Fonte: ABEGÁS. Associação Brasileira das Empresas Distribuidoras de Gás Canalizado

Uma barreira para a utilização do gás natural está associada à incerteza da evolução dos preços devido à instabilidade política de países vizinhos como a Bolívia, o qual tem uma participação significativa no fornecimento de gás natural ao mercado brasileiro.

Em relação ao crescimento, a indústria brasileira de gás natural vem crescendo ano a ano, conforme indicado na Figura 1. Segundo dados consolidados, em meados dos anos 90 a 
participação do gás natural na matriz energética do país não passava dos $3,1 \%$ e hoje o insumo triplicou sua participação e já atinge 9,4\% (ABEGAS, 2007).

No Estado de Mato Grosso do Sul, o setor comercial foi o que mais cresceu entre os segmentos de consumo, sendo que de janeiro a maio de 2005 o consumo aumentou de 94.428 $\mathrm{m}^{3}$ para $211.598 \mathrm{~m}^{3}$ no mesmo período de 2006 (aumento de 140,84\%). A elevação é atribuída ao crescimento do número de clientes, através da ampliação dos ramais de distribuição e as altas tarifas de energia elétrica praticadas no estado.

Na figura 2 tem-se o gráfico do consumo de gás natural residencial e comercial no Estado de Mato Grosso do Sul em metros cúbicos no ano de 2006.

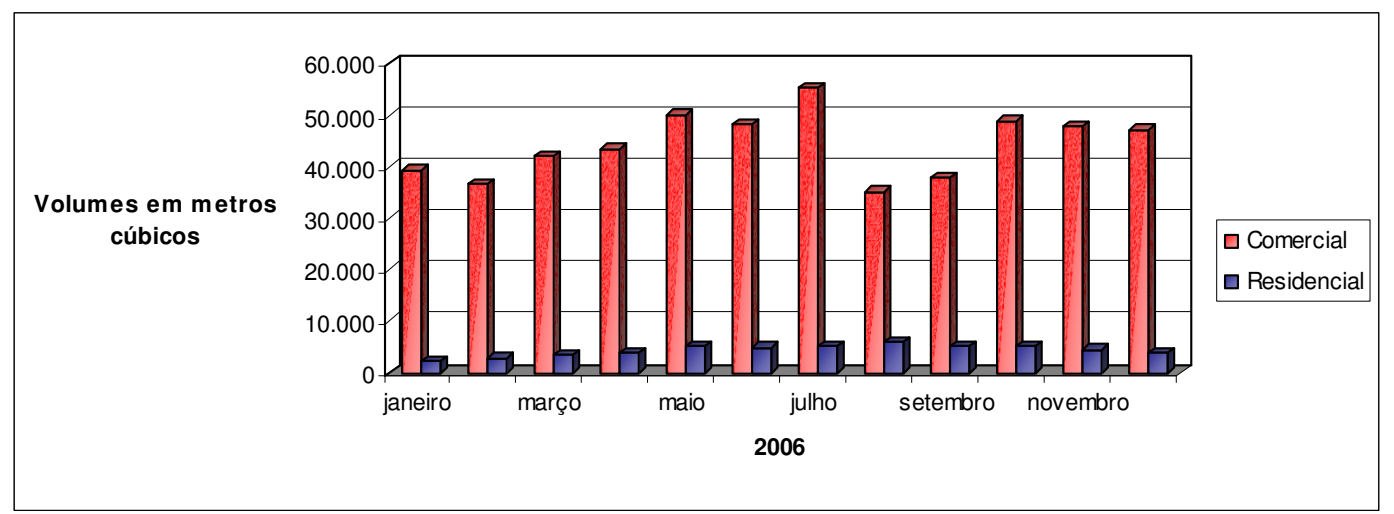

Figura 2 - Consumo de gás natural residencial e comercial em 2006

Fonte: MSGÁS. Companhia de Gás de Mato Grosso do Sul.Informativos

Neste contexto, o aumento do mercado de gás natural nos setores residencial e comercial, incentiva à utilização do GN e, conseqüentemente, incentiva a aplicação de sistemas de cogeração através da microturbina para a geração de energia elétrica e térmica (vapor).

\section{SISTEMA DE COGERAÇÃO}

A cogeração é a tecnologia atualmente disponível de melhor desempenho energético e, portanto, recebe maior estímulo nas politicas energéticas (LORA \& HADDAD, 2006).

Quando se propõe a instalação de um sistema de cogeração, a primeira dúvida que surge diz respeito ao tipo de ciclo termodinâmico que se deve adotar. Como critério inicial deve-se considerar os parâmetros relacionados à capacidade instalada de geração elétrica e o 
nível de temperatura do processo que utiliza a energia térmica (ex: vapor).

Cada vez mais os sistemas de cogeração vem se firmando como uma das tecnologias mais recomendáveis voltada à conservação de energia por sua condição operacional. Para tal, a construção das centrais de cogeração utilizam a combinação de turbinas a gás (ou motores alternativos) e caldeiras de recuperação para aproveitamento do calor de exaustão. A vantagem principal da introdução dessas unidades é a capacidade de redução de custos operacionais e recuperação energética (pelo uso de energia em cascata), tornando-se possível, desta maneira, aumentar a eficiência total de utilização do combustível, que relaciona a quantidade de energia bruta disponível e a quantidade líquida efetivamente aproveitável).

A microturbinas podem ser usadas como reserva, para melhorar a qualidade, garantir a confiabilidade da rede, atender cargas de pico e aplicações adicionais de cogeração. Além disso, as microturbinas para aplicações com combustíveis residuais e gás estão sendo desenvolvidas (LORA \& HADDAD, 2006). Com potências que variam entre $25 \mathrm{~kW}$ e 500 $\mathrm{kW}$, estas microturbinas são adequadas para aplicações comerciais, tais como restaurantes e hotéis, além de pequenos escritórios e condomínios residenciais, dentre outros.

Os sistemas de cogeração geram energia elétrica (ou mecânica) e energia térmica. Esta última corresponde ao calor/frio de processo em prédio comercial e/ou residencial, a partir da queima de um combustível. Essa tecnologia é uma das alternativas mais eficazes para uma utilização consistente e racional da energia primária disponível, principalmente se comparada a centrais térmicas convencionais. Na cogeração pode-se alcançar um aproveitamento de até 80\% (LORA \& HADDAD, 2006) de energia contida no combustível e conseqüentemente, os impactos ambientais associados ao processo de conversão de energia de um modo geral são minimizados. Este impacto é ainda mais reduzido quando se utiliza sistemas movidos à gás natural, os quais apresenta menor nível de poluição atmosférica em relação à outros combustíveis.

$\mathrm{Na}$ Figura 3, tem-se a planta de cogeração mostrando os equipamentos para gerar energia elétrica e vapor, apresentando a microturbina acoplada a uma máquina de refrigeração por absorção (chiller), a qual tem a função de produzir água gelada para refrigeração. 


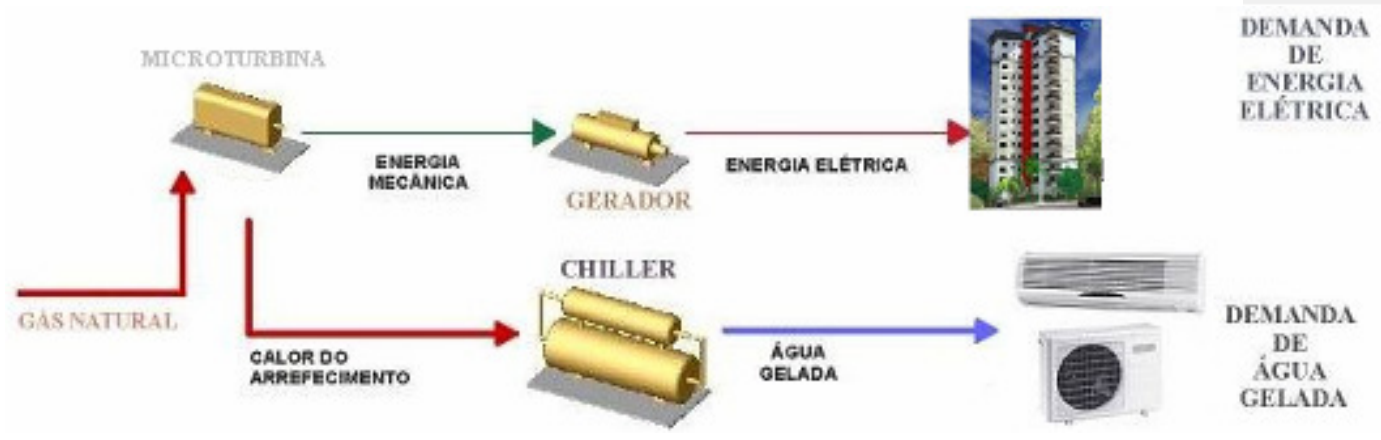

Figura 3 - Exemplo de uma Planta de cogeração

Fonte: COGEN (2001)

Para mensurar a potência da microturbina utilizada no edificio, foram feito os seguintes levantamentos: o edificio possui dois blocos residênciais com um consumo mensal de cerca de $7080 \mathrm{kWh}$, sendo que a área comercial apresenta consumo mensal de $5040 \mathrm{kWh}$, resultando num consumo mensal de $11.880 \mathrm{kWh}$.

Considerando um período de 720 horas mensais de trabalho e um fator de carga - FC de 0,65 , estima-se que a potência mínima adotada para o sistema de cogeração utilizando uma microturbina com uma máquina de refrigeração por absorção na saída dos gases a ser implantado nestas instalações será de $25 \mathrm{~kW}$. Observa-se que o índice do fator de carga permite verificar o quanto que a energia elétrica é utilizada de forma racional e calculado através da razão entre a demanda média, durante um mês, e a demanda máxima registrada no mesmo período).

\section{DINÂMICA DE SISTEMAS}

De acordo com Forester (1968), as idéias relacionadas à Dinâmica de Sistemas (DS) surgiram incialmente na década de 60, com o trabalho de Jay Forrester no MIT (Massachussetts Institute of Technology). Sua teoria foi baseada em sistemas de realimentação e controle com a finalidade e avaliar negócios e contextos organizacionais e sociais.

Uma visão do que representa DS pode ser dita como aquela que tem a sua ênfase na estrutura e no processo de um sistema, assumindo que essa é a melhor maneira que o comportamento dinâmico no "mundo real" pode ser caracterizado ou representado. Então, a 
DS considera o comportamento como sendo a causa principal da estrutura existente. Isto consiste não somente em aspectos físicos de plantas e processos de produção, mas também faz referências importantes a políticas e tradições, que dominam as tomada de decisões.

Essas idéias pode ser transmitidas para um contexto organizacional. Muitas situações sociais e de negócios podem ser caracterizadas pela complexidade de seus elementos de estrutura e de laços e realimentação multicausal que existe entre os referidos elementos.

De maneira geral, o primeiro passo ao utilizar a DS é entender os mecanismos de realimentação que fazem parte do sistema em estudo. Uma maneira de representar tais mecanismos é através de Diagramas de Laço Causal (DLC), também conhecidos como diagramas de influência (FORESTER, 1968).

DLC representam as relações de causa e efeito entre os elementos de um sistema e facilitam a representação do problema real, o desenvolvimento dos modelos matemáticos, a apresentação de resultados de simulação, o desenvolvimento de novas políticas, entre outras.

Uma relação de causa e efeito indica a influência que uma variável exerce sobre outra, isto é, quando é analisada uma relação causal, o efeito de qualquer outra variável é ignorado. Uma relação causal é representada por uma seta unindo duas variáveis, a variável na cauda da seta é a variável causal e a variável na ponta da seta é a variável afetada.

Se uma mudança na direção da variável causal provoca uma alteração na mesma direção da variável afetada, então se diz que a influência é positiva (+), caso contrário, diz-se que é negativa (-). Estes sinais indicativos estão presentes nas figuras apresentadas no item metodologia.

\section{METODOLOGIA}

Este artigo descreve a influência de diversos fatores no contexto da tomada de decisão da cogeração no setor predial residencial e comercial.

Identificando as variáveis de interesse, será possível apresentar uma proposta de modelagem baseada na técnica de DS, permitindo a simulação, análise e discussão de problemas complexos (problemas de difícil formulação matemática ou com grande espaço de busca) e não lineares, formando uma via importante para enfocar e tomar decisões concernentes ao problema.

A análise de viabilidade econômico-financeira baseia-se na comparação dos custos de 


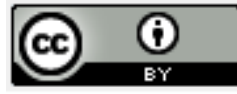

investimentos da utilização do gás natural, comparado ao uso da energia elétrica em prédios residencias e comerciais, analisando os efeitos da competição entre fontes de energia diversas junto aos usuários finais (consumidores).

Para selecionar os modelos de cogeração mais adequados nos setores considerados, optou-se por realizar estudos de viabilidade técnica e econômica em um edificio, escolhido de forma que suas características fossem representativas do setor em questão. Um critério importante para a seleção do edificio foi a existência de demanda por vapor, de forma que, por exemplo, um edifício (prédio) sem um sistema de ar condicionado não poderia ser considerado. Também é importante que a parte produtiva do edificio trabalhe o mais próximo possível de um regime de operação contínuo, pois isto melhora o desempenho econômico da planta de cogeração.

Foram levantados os fatores determinantes para a elaboração da ferramenta de apoio aos novos consumidores residenciais e comerciais, permitindo assim, a elaboração do DLC, ilustrado na Figura 5.

Na Figura 5 tem-se o diagrama utilizado que apresenta as relações de causa e efeito para as variáveis consideradas inicialmente, como determinantes no uso do gás natural em instalações prediais residenciais e comerciais. Tais relações de causa e efeito, servem de base para a criação de um ambiente de simulação que permita ao usuário a tomada de decisão, considerando a influência de múltiplos critérios qualitativos e quantitativos.

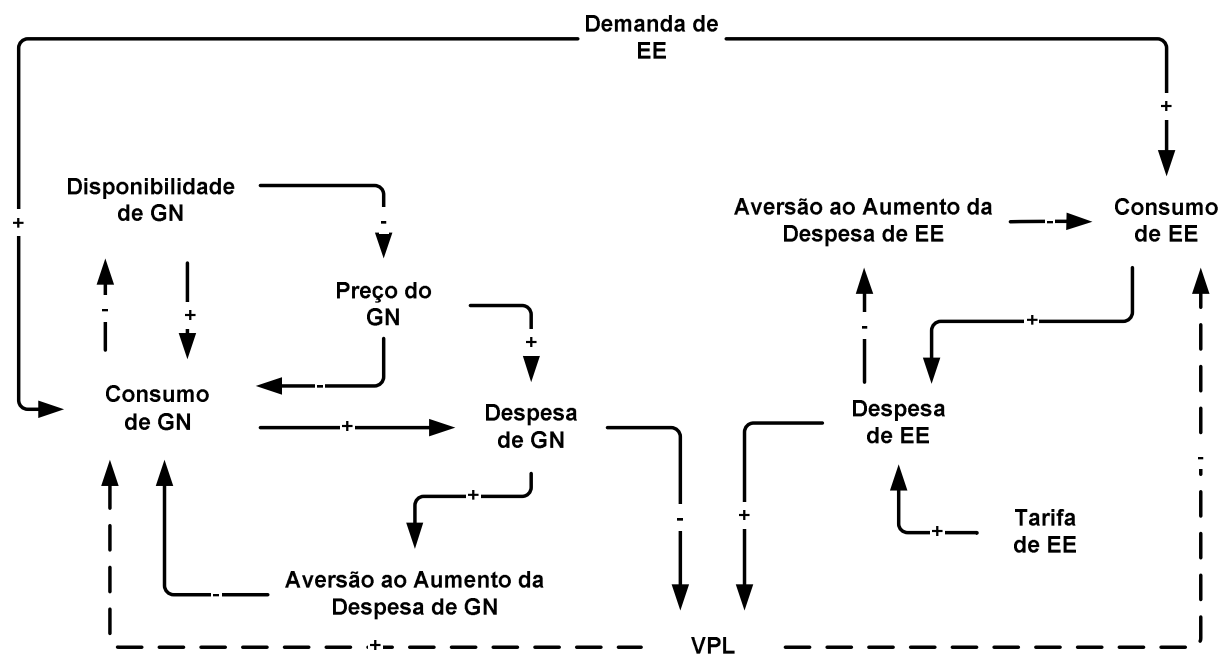

Figura 5 - DLC do uso do gás natural em predios residenciais e comerciais

Fonte: Autores 
Para melhor entender os laços de realimentação e a própria dinâmica do sistema, os laços de realimentação são analisados individualmente na figura 5 (a) e (b).

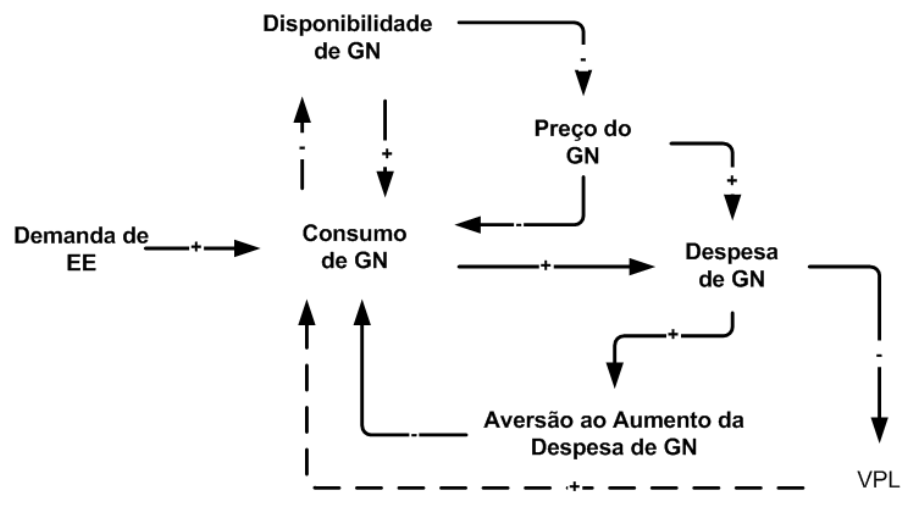

Figura 5 (a) - Mecanismo de Realimentação via Demanda de Energia Elétrica para uso do gás natural.

Fonte: Autores

O DLC enfocando a utilização do gás natural mostra que a tomada de decisão envolve laços de realimentação com dinâmicas diferentes. Ou seja, investimentos na demanda de energia elétrica resultam, conforme mostra Figura 5 (a) em melhoria do fornecimento deste energético, e no consequiênte aumento do consumo de gás natural, conforme indicado nas setas com (+) da figura. Portanto, o aumento no consumo implica, num aumento do montante de gás contratado, resultando assim, na possibilidade de obter melhores preços junto ao fornecedor de energia primária, o que tem influência positiva na despesa de GN.

Por outro lado, a figura 5 (b) mostra que com o aumento da demanda de energia elétrica (EE), eleva o consumo desta fonte de energia, e num consequente aumento das despesas com o uso da energia elétrica. Desta forma, a relação entre o aumento da demanda de energia elétrica e a aversão ao aumento das despesas de energia elétrica passa a ser uma variável de decisão com influência direta no consumo de energia elétrica.

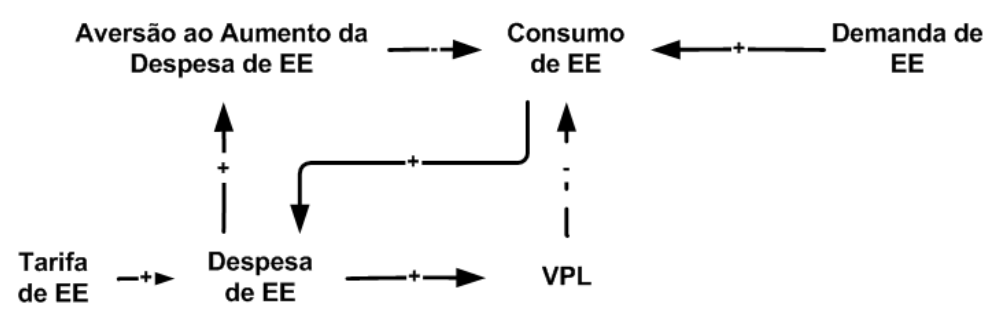

Figura 5 (b) - Mecanismo de Realimentação via Demanda de Energia Elétrica para uso da energia elétrica

Fonte: Autores 
É importante ressaltar, que o DLC foi contruído em função do conhecimento relativo ao sistema, onde, por muitas vezes, resulta da subjetividade e intuição do modelador, neste caso os autores deste trabalho.

\section{CONCLUSÃO}

Este trabalho apresenta uma metodologia de analise econômico-financeira da aplicação de sistemas de cogeração utilizando o gás natural em instalações prediais residencial e comercial.

A utilização do gás natural como alternativa à EE é avaliada segundo a ótica da DS através da identificação das variáveis de interesse e da elaboração das relações de influência entre os parâmetros do sistema na modelagem do problema.

A importância desta metodologia na modelagem de sistemas de cogeração de energia consiste em permitir avaliar a influência, positiva (+) ou negativa (-), de fatores tais como: o crescente aumento nas tarifas de energia elétrica, associada à perspectiva de retomada do crescimento econômico e à incerteza quanto à expansão da oferta de eletricidade e GN, na viabilidade destes sistemas nos setores comerciais e residenciais.

\section{REFERÊNCIAS}

AMBIENTE BRASIL (2007). In: Informações de Gás Natural. Disponível em: http://www.ambientebrasil.com.br/composer.php3?base=./energia/index.html\&conteudo=./en ergia/gasnatural.html. Acesso em: 18 abril 2007.

ANEEL (2007). In: Informações Agência Nacional de Energia Elétrical. Disponível em: http://www.aneel.gov.br/. Acesso em: 18 abril 2007.

COGEN (2001). A guide to cogeneration. European Association for the Promotion of Cogeneration. Bélgica.

ENERGIA (2007). In: Informações de Energia Elétrica. Disponível em: http://www.sanegas.com.br. Acesso em: 2 maio 2007.

FORRESTER, J. W. Industrial Dinamics. Management Science, 14, No, 7; May 1968.

LORA, E. E. S. \& HADDAD, J. Geração Distribuída: Aspectos Tecnológicos, Ambientais e Institucionais Vol. 1, p.3. Rio de Janeiro: Interciência, 2006. 


\section{Revista Produç@o @o}

Associação Brasileira de Engenharia de Produção - ABEPRO

Universidade Federal de Santa Catarina - UFSC

www.producaoonline.org.br

ISSN 1676 - 1901 / Vol. IX/ Num.II / 2009

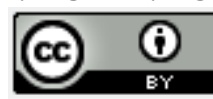

MSGÁS (2007). In: Informações de Gás Natural. Disponível em: http://www.msgas.com.br/imprensa.html. Acesso em: 18 abril 2007.

ORTEGOSA, C. Z. Metodologia para estudos da viabilidade econômico-financeira no uso do gás natural em instalações prediais residenciais: uma abordagem via dinâmica de sistemas. Dissertação de mestrado. Universidade Federal de Mato Grosso do Sul. Fev, 2006.

PAC (2007). In: Informações do Programa de Aceleração do Crescimento. Disponível em: http://www.dieese.org.br/notatecnica/notatec43PACehidrocarbonetos.pdf. Acesso em: 18 abril 2007.

PAC (2007). In: Informações relacionados a cogeração. Disponível em: http://www.br.com.br. Acesso em: 06 outubro 2008. 